\title{
CONTINUITY OF SYSTEMS OF DERIVATIONS ON F-ALGEBRAS
}

\section{R. L. CARPENTER ${ }^{1}$}

Abstract. Let $A$ be a commutative semisimple $F$-algebra with identity, and let $D_{0}, D_{1}, \cdots$ be a system of derivations from $A$ into the algebra of all continuous functions on the spectrum of $A$. It is shown that the transformations $D_{0}, D_{1}, \cdots$ are necessarily continuous. This result is used to obtain a characterization of derivations on $\mathrm{Hol}(\Omega)$ where $\Omega$ is an open polynomially convex subset of $\mathbf{C}^{n}$.

Introduction. In [4] Gulick proves that if $D_{0}, D_{1}, \cdots, D_{n}$ is a system of derivations from a commutative semisimple regular $F$ algebra into $C(S(A))$, the algebra of all continuous functions on the spectrum $S(A)$ of $A$, then the functions $D_{0}, D_{1}, \cdots, D_{n}$ are all continuous. This theorem is not as general as might be hoped for, since the regularity condition is far too restrictive. It precludes, for example, the existence of analytic structure in the algebra, while algebras of analytic functions are among the most interesting algebras which have derivations. It is shown in this paper that the regularity condition is superfluous. We use a lemma proved by Johnson [5] and a technique we developed in [2] to prove the more general theorem that if $A$ is a commutative semisimple $F$-algebra and $D_{0}$, $D_{1}, \cdots$ is a system of derivations from $A$ into $C(S(A))$, then each of the $D_{i}, i=0,1, \cdots$, is necessarily continuous. This theorem allows us to characterize derivations on $\operatorname{Hol}(\Omega)$ for $\Omega$ an open polynomially convex subset of $\boldsymbol{C}^{n}$.

Preliminaries. An $F$-algebra is an algebra over the complex numbers which is a complete $T_{2}$ topological space with respect to a topology determined by a countable family of multiplicative seminorms $\left\{\|\cdot\|_{i}\right\}, i=1,2, \cdots$. No generality is lost if the seminorms are assumed to be increasing. That is, we may assume $\|\cdot\|_{i} \leqq\|\cdot\|_{i+1}$ for $i=1,2, \cdots$. In this paper we will always assume that the algebras under consideration are commutative and have identities. The

Presented to the Society, January 24, 1971 under the title Systems of derivations and algebras of functions; received by the editors December 2, 1970.

AMS 1969 subject classifications. Primary 4650.

Key words and phrases. F-algebra, derivation, systems of derivations, continuity of derivations.

1 The author would like to thank the referee for pointing out an error in the original proof of Theorem 5 . 
spectrum $S(A)$ of an $F$-algebra $A$ is the space of all continuous homomorphisms of the algebra $A$ on to the complex numbers. $S(A)$ is given the Gelfand topology. If $A$ is an $F$-algebra, then $A$ can be realized as the inverse limit of a sequence of Banach algebras $A_{n}$ (see [6]). There is a natural homeomorphism of the spectrum $S\left(A_{n}\right)$ onto a compact subset of $S(A)$. We will henceforth identify $S\left(A_{n}\right)$ with its image in $S(A)$; with this identification, $S(A)$ is the union of the compact sets $S\left(A_{n}\right)$ (see [1], [6]). For an element $f$ of $A$ we will use $f^{\wedge}$ to denote the Gelfand transform of $f$. That is, $f^{\wedge}$ is the function defined on $S(A)$ by $f^{\wedge}(\phi)=\phi(f)$ for every $\phi$ in $S(A)$. An $F$-algebra $A$ is semisimple iff for an element $f$ of $A, \phi(f)=0$ for every $\phi \in S(A)$ implies $f=0$ (see [6]).

We will use the symbol $\boldsymbol{C}$ to denote the complex numbers. For a topological space $T, C(T)$ will denote the algebra of all complex valued continuous functions on $T$.

Systems of derivations. For the rest of this paper, $A$ will denote a semisimple commutative $F$-algebra with identity, and $S(A)$ will denote the spectrum of $A$. We fix a sequence $A_{1}, A_{2}, \cdots$ of Banach algebras such that $A$ is the inverse limit of the algebras $A_{i}$. A collection $D_{0}, D_{1}, \cdots$ of linear maps from $A$ into $C(S(A))$ is called a system of derivations on $A$ iff $D_{0} f=f^{\wedge}$ for every $f$ in $A$, and, for any $f, g$ in $A$,

$$
D_{k}(f g)=\sum_{i=0}^{k}\left(\begin{array}{l}
k \\
i
\end{array}\right) D_{i} f D_{k-i} g
$$

for each $k=1,2, \cdots$. For a detailed discussion of systems of derivations, the reader is referred to [4].

LEMma 1 (Rosenfeld [7]). If $\phi \in S(A)$ is isolated in each $S\left(A_{n}\right)$ which contains it, then $\phi$ is isolated in $S(A)$.

Lemma 2 (Johnson [5]). Let $\phi_{1}, \phi_{2}, \cdots$ be a sequence of distinct points in $S(A)$. For each positive integer $k$, there is $a y_{k} \in A$ such that $\phi_{i}\left(y_{k}\right)=0$ for $i<k$ and $\phi_{i}\left(y_{k}\right) \neq 0$ for $i \geqq k$.

Proof. For $F$-algebras this lemma admits an easier proof than the one originally given by Johnson. The reader is referred to [2] for the proof.

For a point $\phi \in S(A)$, let $h_{\phi}$ denote the functional on $C(S(A))$ defined by $h_{\phi}(f)=f(\phi)$ for every $f \in C(S(A))$.

Lemma 3. Suppose that $A$ is a semisimple F-algebra, that $\phi$ is an 
isolated point of $S(A)$, and that $D_{0}, D_{1}, \cdots$ is a system of derivations on $A$. Then for $i=1,2, \cdots$ we have that $h_{\phi} D_{i}$ is the zero functional on $A$.

Proof. The Shilov idempotent theorem implies there is an $e \in A$ such that $e^{\wedge}(\psi)=1$ for $\psi \in S(A)-\{\phi\}$ and $e^{\wedge}(\phi)=0$. Since $A$ is semisimple $e$ is an identity for the ideal $I=\operatorname{ker} \phi$.

The functional $h_{\phi} D_{1}$ is a point derivation at $\phi$ and consequently $I^{2}+C$ is contained in kernel $\left(h_{\phi} D_{1}\right)$. Since $e$ is an identity for $I$, we have $I^{2}=I$. Therefore $h_{\phi} D_{1}=0$.

Fix a positive integer $k$, and assume that $h_{\phi} D_{1}, \cdots, h_{\phi} D_{k}$ are zero. For any $f \in I$, we have

$$
h_{\phi} D_{k+1}(f)=h_{\phi} \sum_{i=0}^{k+1}\left(\begin{array}{c}
k+1 \\
i
\end{array}\right) D_{i}(e) D_{k+1-i}(f)=0 .
$$

For an arbitrary $f \in A$, we have $f=g+\alpha$ where $g \in I$ and $\alpha \in C$. An easy induction proof shows that $D_{j}(\alpha)=0$ for any $\alpha \in C$ and $j=1$, $2, \cdots$ Therefore $h_{\phi} D_{k+1}=0$, and induction implies $h_{\phi} D_{1}, h_{\phi} D_{2}, \cdots$ are all zero.

Let $y \in A, \phi \in S(A)$, and $D_{0}, D_{1}, \cdots$ be a system of derivations on A.

Lemma 4. Suppose $\phi(y)=0$; fix a positive integer $k$. Then for any $j<k$ we have $h_{\phi} D_{j} y^{k}=0$.

Proof. It is clear that $h_{\phi} D_{0} y=0$.

Fix a positive integer $n$ and suppose that $h_{\phi} D_{j} y^{n}=0$ for $j<n$. Let $j<n+1$. Then

$$
h_{\phi} D_{j} y^{n+1}=h_{\phi} \sum_{i=0}^{j}\left(\begin{array}{l}
j \\
i
\end{array}\right) D_{i} y D_{j-i} y^{n}=h_{\phi}\left(D_{0} y D_{j} y^{n}\right)=0 .
$$

The lemma now follows from the axiom of induction.

Theorem 5. Let $A$ be a semisimple F-algebra with identity and $D_{0}$, $D_{1}, \ldots$ be a system of derivations from $A$ into $C(S(A))$ such that $D_{0} f=f^{\wedge}$ for every $f \in A$. Then each of the derivations $D_{i}, i=0,1, \cdots$, is continuous.

Proof. It is clear that $D_{0}$ is continuous. Fix a positive integer $k$, and assume that $D_{i}$ is continuous for $i<k$. We will use the closed graph theorem to show that $D_{k}$ is continuous. However, $C(S(A))$ is complete with respect to the compact open topology if and only if $S(A)$ is a $k$-space, and it has recently been shown that there are $F$-algebras $B$ for which $S(B)$ is not a $k$-space (see [3]). In order to 
apply the closed graph theorem, we regard $D_{k}$ not as a function into $C(S(A))$ but rather as a function into $C(k(S(A)))$. Where $k(S(A))$ is the set $S(A)$ with the weak topology generated by the Gelfand compact subsets of $S(A)$. The space $C(k(S(A)))$ is complete with respect to the compact open topology. Hence, we can conclude $D_{k}: A$ $\rightarrow C(k(S(A)))$ is continuous if we can show its graph is closed; this will imply $D_{k}$ is continuous when regarded as a function into $C(S(A))$.

In order to show that the graph of $D_{k}$ is closed, we consider the set $T=\left\{\phi \in S(A): h_{\phi} D_{k}\right.$ is continuous $\}$.

Let $\phi_{0}$ be an isolated point in $S(A)$. It follows from Lemma 3 that $\phi_{0}$ is in $T$. Hence, $T$ contains all of the isolated points of $S(A)$.

Let $\phi_{0}$ be an element of $S(A)$ and suppose $\phi_{0}$ is not isolated in $S(A)$; Lemma 1 implies there is an integer $n$ such that $\phi_{0}$ is not isolated in $S\left(A_{n}\right)$. If $\phi_{0}$ is not a limit point of $S\left(A_{n}\right) \cap T$, then we can choose a sequence $\phi_{1}, \phi_{2}, \ldots$ of distinct points in $S\left(A_{n}\right)-T$.

Use Lemma 2 to select a sequence $\left\{y_{i}\right\} \subset A$ such that $\phi_{i}\left(y_{j}\right)=0$ for $i<j$ and $\phi_{i}\left(y_{j}\right) \neq 0$ for $i \geqq j$. Making use of the fact that $h_{\phi_{i}} D_{k}$ is not continuous at zero and that $D_{j}$ is continuous for $j<k$, we choose a sequence $\left\{x_{i}\right\} \subset A$ such that

$$
\begin{aligned}
\max \left\{\left\|x_{i}\left(y_{1} \cdots y_{i}\right)^{k+1}\right\|_{i}, \| x_{i}\left(y_{1} \cdots y_{j-1} y_{j+1}\right.\right. & \left.\cdots y_{i}\right)^{k+1} \|_{i} \\
j & =2, \cdots, i-1\}<2^{i}
\end{aligned}
$$

and

$$
\begin{aligned}
h_{\phi_{i}} D_{k} x_{i} \geqq & {\left[i+\left|h_{\phi_{i}} D_{k} \sum_{j=0}^{i-1} x_{j}\left(y_{1} \cdots y_{j}\right)^{k+1}\right|\right.} \\
& \left.+\left|h_{\phi_{i}} \sum_{j=1}^{k}\left(\begin{array}{c}
k \\
j
\end{array}\right) D_{j}\left(y_{1} \cdots y_{i}\right)^{k+1} D_{k-j} x_{i}\right|\right]\left|\phi_{i}\left(y_{1} \cdots y_{i}\right)\right|^{-k-1} .
\end{aligned}
$$

The symbol $\|\cdot\|_{i}$ denotes the $i$ th seminorm on $A$, and we assume the seminorms are increasing.

The series $\sum_{i=1}^{\infty} x_{i}\left(y_{1} \cdots y_{i}\right)^{k+1}$ converges to an element $x$ of $A$. For any positive integer $i$, we have

$$
\begin{aligned}
h_{\phi_{i}} D_{k} x= & h_{\phi_{i}} D_{k} \sum_{j=1}^{i-1} x_{j}\left(y_{1} \cdots y_{j}\right)^{k+1}+h_{\phi_{i}} D_{k} x_{i}\left(y_{1} \cdots y_{i}\right)^{k+1} \\
& +h_{\phi_{i}} D_{k} \sum_{j=i+1}^{\infty} x_{j}\left(y_{1} \cdots y_{j}\right)^{k+1}
\end{aligned}
$$

The expression $h_{\phi_{i}} D_{k} \sum_{j=i+1}^{\infty} x_{j}\left(y_{1} \cdots y_{j}\right)^{k+1}$ may be written as 


$$
h_{\phi_{i}} \sum_{l=0}^{k}\left(\begin{array}{l}
k \\
l
\end{array}\right) D_{l} y_{i+1}^{k+1} D_{k-l} \sum_{j=i+1}^{\infty} x_{j}\left(y_{1} \cdots y_{i} y_{i+2} \cdots y_{j}\right)^{k+1} .
$$

Using Lemma 4, we see that this expression is zero. Hence

$$
\begin{aligned}
\left|h_{\phi_{i}} D_{k} x\right|=\mid h_{\phi_{i}} D_{k} \sum_{j=1}^{i-1} x_{j}\left(y_{1} \cdots y_{j}\right)^{k+1}+\left(h_{\phi_{i}} D_{k} x_{i}\right) \phi_{i}\left(y_{1} \cdots y_{i}\right)^{k+1} & \\
& +h_{\phi_{i}} \sum_{j=1}^{k}\left(\begin{array}{c}
k \\
j
\end{array}\right) D_{j}\left(y_{1} \cdots y_{i}\right)^{k+1} D_{k-j} x_{i} \mid>i .
\end{aligned}
$$

This shows that the function $D_{k} x$ is unbounded on $S\left(A_{n}\right)$. But this is a contradiction, since $D_{k} x$ is a continuous function on $S\left(A_{n}\right)$ and $S\left(A_{n}\right)$ is compact. Hence $\phi_{0}$ must be a limit point of $T \cap S\left(A_{n}\right)$.

We thus have that for each $\phi \in S(A)$ there is an integer $n$ such that $\phi$ is in the closure of $T \cap S\left(A_{n}\right)$. Since each $f \in C(k(S(A)))$ is continuous when restricted to $S\left(A_{n}\right)$, we have that $\left\{h_{\phi}: \phi \in T\right\}$ separates the points of $C(k(S(A)))$. This is sufficient to guarantee that the graph of $D_{k}$ is closed. Hence $D_{k}$ is continuous. Induction implies $D_{i}$ is continuous for each $i=1,2, \cdots$.

By a derivation on the algebra $A$, we mean a linear function $D$ from $A$ into $C(S(A))$ such that $D(f g)=f^{\wedge} D g+g^{\wedge} D f$ for any $f, g \in A$. Now suppose $D$ is a derivation on $A$, and $x_{1}, \cdots, x_{n}$ are elements of $A$, and $p$ is a polynomial in $n$ variables. An elementary calculation shows that $D p\left(x_{1}, \cdots, x_{n}\right)=\sum_{i=1}^{n}\left(D x_{i}\right) d_{i} p\left(x_{1}, \cdots, x_{n}^{\hat{n}}\right)$. Here $d_{i}$ denotes the partial derivative of $p$ with respect to the $i$ th variable.

Consider the algebra $\mathrm{Hol}(\Omega)$ for a polynomially convex open subset $\Omega$ of $\boldsymbol{C}^{n}$. (Hol $(\Omega)$ denotes the algebra of all analytic functions on $\Omega$ with the compact open topology.) Since $\Omega$ is polynomially convex, polynomials in the coordinate functions $z_{1}, \cdots, z_{n}$ are dense in $\operatorname{Hol}(\Omega)$. Let $D$ be a derivation from $\operatorname{Hol}(\Omega)$ into $C(\Omega)$. For any polynomial $p$, we have $D p\left(z_{1}, \cdots, z_{n}\right)=\sum_{i=1}^{n} D z_{i} d_{i} p\left(z_{1}, \cdots, z_{n}\right)$. If $f \in \operatorname{Hol}(\Omega)$, we can choose a sequence $\left\{p_{j}\right\}$ of polynomials such that $\left\{p_{j}\right\}$ converges to $f$ with respect to the compact open topology. For each $i=1, \cdots, n$, we have $\left\{d_{i} p_{j}\right\}_{j}$ converges to $d_{i} f$. Theorem $5 \mathrm{im}$ plies that $D$ is continuous. Therefore $D f=\sum_{i=1}^{n} D z_{i} d_{i} f$. We thus have the following theorem.

TheOREM 6. If $\Omega$ is an open polynomially convex subset of $\boldsymbol{C}^{n}$ and $D$ is a derivation from $\operatorname{Hol}(\Omega)$ into $C(\Omega)$, then $D f=\sum D z_{i} d_{i} f$ for every $f \in \operatorname{Hol}(\Omega)$.

We note that a similar characterization is valid for systems of derivations on $\mathrm{Hol}(\Omega)$, since Theorem 5 guarantees the continuity of any such system. 


\section{REFERENCES}

1. R. Arens, Dense inverse limit rings, Michigan Math. J. 5 (1958), 169-182. MR $21 \# 3780$.

2. R. L. Carpenter, Uniqueness of topology for commutative F-algebras, Proc. Amer. Math. Soc. (to appear).

3. A. G. Dors, On the spectrum of an F-algebra, Thesis, University of Utah, Salt Lake City, Utah, 1970.

4. F. Gulick, Systems of derivations, Trans. Amer. Math. Soc. 149 (1970), 465-488.

5. B. E. Johnson, Continuity of derivations on commutative algebras, Amer. J. Math. 91 (1969), 1-10. MR 39 \#7433.

6. E. A. Michael, Locally multiplicatively-convex topological algebras, Mem. Amer. Math. Soc. No. 11 (1952). MR 14, 482.

7. M. Rosenfeld, Commutative F-algebras, Pacific J. Math. 16 (1966), 159-166. MR 32 \#8196.

University of Houston, Houston, Texas 77004 